\title{
The Validity of the Alcohol-Use-Disorder-Identification-Test and a Novel Proxy Test in the Assessment of Alcohol Consumption in the Cognitively Impaired Elderly
}

\author{
Anesh Patel ${ }^{1,5}$, Luke Gibbor ${ }^{1}$, Rebecca Douch ${ }^{1}$, Miles Welstead ${ }^{2}$, Emma Cahill ${ }^{2}$, Christos Kouimtsidis ${ }^{4,5}$, Danielle \\ Wilson ${ }^{1}$, Sarah Gregory ${ }^{1}$, Merrie Manalo ${ }^{1}$, Prem Mandalia ${ }^{1}$, Stephanie Deriziotis ${ }^{1}$, Seyda Kaya ${ }^{1}$, Katie Wells ${ }^{5}$ and \\ Vanessa Raymont ${ }^{2,3,5 \star}$
}

${ }^{1}$ West London Mental Health Trust, Isleworth, UK

${ }^{2}$ Centre for Dementia Prevention, University of Edinburgh, UK

*Corresponding author

Vanessa Raymont, University of Oxford, Department of Psychiatry, Warneford Hospital, Oxford OX3 7JX, UK, Tel: 01865618232; E-mail:

Vanessa.raymont@psych.ox.ac.uk

${ }^{4}$ Surrey and Borders Partnership NHS Foundation Trust, UK

Submitted:01 May2018; Accepted:07 May2018; Published: 17 May 2018

Imperial College London, UK

\begin{abstract}
Aims: Alcohol is consumed excessively by almost a quarter of the population. A report by highlighted underdiagnosis of alcohol misuse in the elderly [1]. There is a lack of research focusing on assessment of alcohol use and its impact in older people. This study aimed to assess the validity of the Alcohol-Use-Disorder-Identification-Test (AUDIT) in an elderly population with cognitive impairment and to establish the validity of a novel adaption of the AUDIT used as a proxy measure.
\end{abstract}

Methods: Opportunistic observational study examining the assessment of alcohol consumption in an elderly population with mild cognitive impairment or mild dementia. The study aimed to evaluate the self-report AUDIT versus a "proxy" AUDIT compared to a plasma Carbohydrate-deficientTransferrin (CDT) level.

Findings: Scores were comparable between the "proxy" AUDIT and the AUDIT. Neither measure of alcohol intake correlated significantly with CDT levels. There was no association between alcohol intake and cognition or quality of life ratings.

Conclusions: We suggest the AUDIT could be a useful screening tool in detecting alcohol misuse in the elderly with cognitive impairment. The "proxy" AUDIT was also found to be a valid screening tool, however further work is needed to confirm these findings.

Keywords: Alcohol-Misuse, Audit, Elderly, Mild Cognitive Impairment,-Cognitive-Impairment,-Dementia,-Cdt, Hazardous Drinking

Background

Alcohol is the most commonly used drug in the UK and its misuse is a growing problem. Up to $24 \%$ of people in the UK consume alcohol in ways that are potentially harmful to their health [2]. Moreover, it has been suggested that $4 \%$ of the population are now alcohol dependent ( $6 \%$ of men and $2 \%$ of women), making it difficult for them to reduce their consumption despite increasingly harmful events [3]. However, the increase in health burden is not confined to the young; Moos found that $49 \%$ percent of men aged $75-85$ and $27 \%$ of women aged $75-85$ were consuming more than two drinks per day or seven drinks per week, which was associated with increased levels of alcohol related problems [4]. Recent research has pointed to a higher rate of alcohol consumption amongst the elderly than previously anticipated. The 2011 report "Our Invisible Addicts" outlined the under-diagnosis of elderly patients misusing alcohol and emphasised the shortage of research into how accurately we identify those at risk [1]. The report highlighted the increase of alcohol misuse and mortality associated with this misuse in our elderly population when compared to the rest of the population. This suggests a potential increase in burden on society and individuals as the population ages, as well as healthcare systems. Accurate screening tools and treatment strategies specifically designed for elderly populations are yet to be developed with little change in public health surveillance since the reports' publication. Although alcohol misuse is one of the fastest growing health issues for older adults, it is less likely to be discussed or screened for in the elderly [5-7]. 
In addition, older adults may be more sensitive to the effects of alcohol which may contribute to the development of many cognitive disorders [8]. Excessive alcohol consumption in later life is seen to exacerbate cognitive decline and lead to a higher risk of developing dementia [9]. 'Prodromal dementia' or mild cognitive impairment (MCI) prevalence ranges from 3-36\% in those aged 75 and over with annual conversion rates to dementia estimated to be approximately $5-10 \%[10,11]$. Thus, with an increasingly elderly population, the prevalence of $\mathrm{MCI}$ is rising, and yet to date, there has been little in the literature about the co-morbidity of MCI and alcohol misuse in the UK. In fact, alcohol misuse and its harmful effects on cognitive ability in older adults is a research field which has been somewhat overlooked $[5,9,12]$. Wadd found that many cases of cognitive impairment in older adults with alcohol problems go undetected using current screening methods [13]. Research has shown that alcohol can accelerate cognitive decline when used in heavy and prolonged bouts however other research has found that light to moderate usage may in fact be protective against decline when compared to alcohol abstainers [14-18]. Peters found that small amounts of alcohol may be protective against Alzheimer's disease but not vascular dementia [19]. Moreover, Chan, Chiu and Chu found that average weekly alcohol consumption was significantly higher in those who had cognitive impairment compared to those who did not in their sample of 314 Southern Chinese people aged 65 and over, suggesting that there may be an association between alcohol consumption and cognitive impairment, although the directionality was unclear. Conversely, Almeida suggested that heavy regular drinking and alcohol abuse were not associated with cognitive impairment in a 6 year follow up study of men aged $60-83[15,20]$. Excessive and prolonged use of alcohol impacts structural and functional brain damage may lead to a condition known as Alcohol Related Dementia (ARD). ARD is a term that has little recognition as a separate entity compared to other causes of dementia, as the links are not yet clearly understood [21-23].

Yet memory impairment can be a particular issue when asking individuals to recall their alcohol usage [13]. Several studies have found that an informant report combined with self-reported screening for dementia was more accurate in the detection of dementia than solely using self-reported measures [24-26]. This may be particularly evident in assessing alcohol usage due to the impaired ability to selfreport due to memory decline or other factors such as anosognosia.
In this study we aimed to examine how we can best identify hazardous alcohol use, defined by a level of drinking which may be causing either physical or mental harm, or alcohol dependence in elderly subjects with cognitive impairment (CI) [27]. Hazardous drinking has been previously identified using screening tools such as the World Health Organisation's AUDIT (Alcohol Use Disorder Identification Test [see Appendix A] [28]. The AUDIT examines drinking related behaviours and harms, as well as frequency and amount of drinking in the past year, and was first validated in six different countries, yielding $92 \%$ sensitivity and $94 \%$ specificity for harmful or hazardous alcohol consumption using a cut off score of 8 $[28,29]$. It has also been validated in a variety of ethnic groups and ages up to 86 years [30,31]. However, with an ageing population and a dramatic rise in patients with MCI and dementia, we questioned whether this screening tool is as reliable in older people with reduced cognitive capabilities. The AUDIT was developed by the World Health Organization (WHO) and has been validated internationally for screening harmful and hazardous alcohol misuse in the elderly, as have the Michigan Alcohol Screening Test - Geriatric version MAST-G; and the Short MAST-G, SMAST-G; [32-38]. While many studies have looked at the link between alcohol and CI, few if any, have robustly assessed the validity of the AUDIT or any other alcohol screening tool in elderly people with CI, who we hypothesise may have trouble accurately recalling their alcohol intake. Rocca did find a $71-75 \%$ agreement on alcohol consumption between those with a neurological condition and their next of kin $[39,40]$. We therefore used a proxy version of the AUDIT given to participants' carers, in order to assess the validity of their report compared to the subject's AUDIT ratings, as well as a gold standard measurement of hazardous drinking; plasma Carbohydrate-deficient transferring (CDT, a quantitative measure of alcohol consumption in the last month) $[41,42]$. We hypothesised that carers may be able to give a more reliable account of patients' alcohol intake, possibly owing to patient under-reporting because of memory deficits associated with CI. One strength of the AUDIT is that it provides guidelines as how to quantify units based on type of drink. Yet, the AUDIT has rarely been used in a proxy manner before; one study utilised it in trauma patients [43]. As well as assessing the validity of these screening methods, we assessed if there was a correlation between alcohol intake and level of CI using the Addenbrooke's Cognitive Examination - Revised [44].

\section{Alcohol Use Disorders Identification Test (AUDIT)}

Please circle the answer that is correct for you.

1. How often do you have a drink containing alcohol?

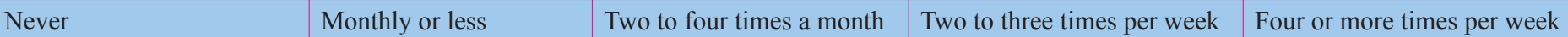

2. How many drinks containing alcohol do you have on a typical day when you are drinking?

\begin{tabular}{|l|l|l|l|l}
1 or 2 & 3 or 4 & 5 or 6 & 7 to 9 & 10 or more
\end{tabular}

3. How often do you have six or more drinks on one occasion?

\begin{tabular}{|l|l|l|l|l} 
Never & Less than monthly & Monthly & Two to three times per week & Four or more times per week
\end{tabular}

4. How often during the last year have you found that you were not able to stop drinking once you had started?

\begin{tabular}{|l|l|l|l|l} 
Never & Less than monthly & Monthly & Two to three times per week & Four or more times per week
\end{tabular}

5. How often during the last year have you failed to do what was normally expected from you because of drinking?

\begin{tabular}{|l|l|l|l|l|}
\hline Never & Less than monthly & Monthly & Two to three times per week & Four or more times per week
\end{tabular}

6. How often during the last year have you needed a first drink in the morning to get yourself going after a heavy drinking session?

\begin{tabular}{|l|l|l|l|l|}
\hline Never & Less than monthly & Monthly & Two to three times per week & Four or more times per week \\
\hline
\end{tabular}




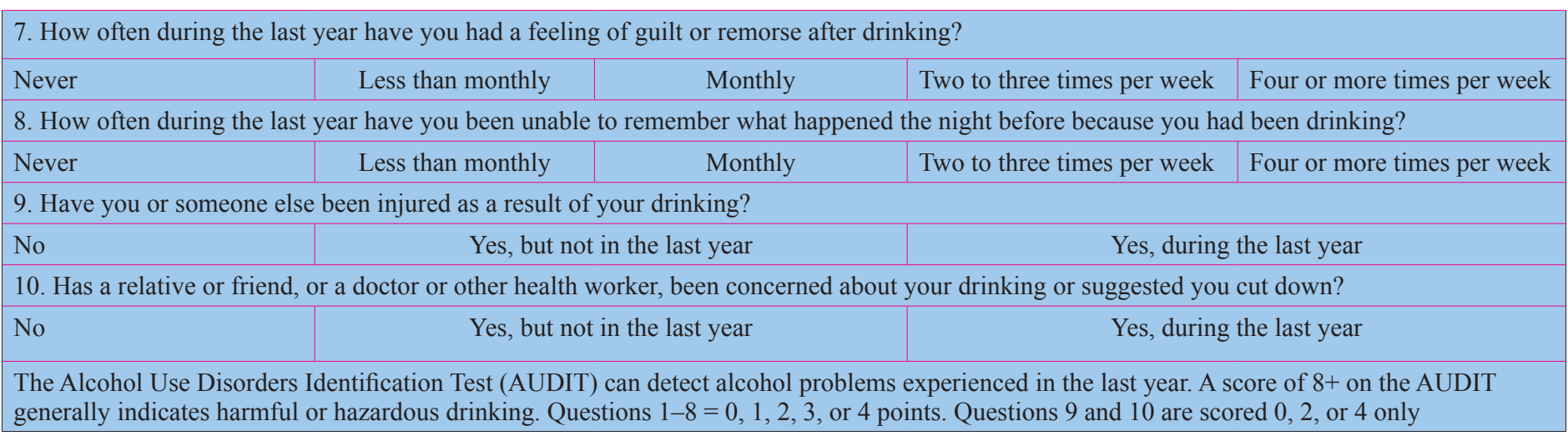

One issue is that if we can successfully identify older people with CI whose drinking is harmful, are we able to offer any beneficial interventions? The relationship between alcohol use and cognitive impairment in older people is complex, and studies thus far have been inconclusive for a number of reasons including; inconsistencies with the time of follow up, heterogeneity of cognitive impairment and the reliability of obtaining accurate information on alcohol consumption [45]. It is clear that alcohol misuse is a significant and growing problem among the elderly and with an increase in the prevalence of mild cognitive impairment, such misuse in those who are cognitively impaired may prove to be a particularly challenging problem to assess and treat. In addition, few studies have examined the wider impact of harmful drinking on quality of life and carer burden, which we assessed using the EQ-5D Health Questionnaire (validated for use in cognitive impairment) and the Neuropsychiatric Inventory (NPI) [46]. We therefore aimed to provide insight into a possible screening tool and/or a proxy screening tool for alcohol misuse in the elderly with cognitive impairment, and assess any correlations between alcohol intake, cognition and quality of life.

\section{Materials and Methods \\ Study Population}

This cross-sectional study was conducted at the West London Cognitive Disorders Treatment and Research Unit, West London Mental Health NHS Trust (WLMHT). Patients attending WLMHT who presented with MCI or mild dementia were invited to take part in the study or were recruited by telephone from a registry of patients who had previously agreed to be contacted about clinical research [46].

\section{Inclusion Criteria}

- Individuals of age above 50 years

- A clinical diagnosis of MCI or mild dementia (MMSE score $>=15$ )

- No other current significant neurological or psychiatric illness

- Capable of giving written informed consent

- Reliable trial partner/caregiver/informant

\section{Exclusion Criteria}

- Unwillingness or inability to follow the procedures required

- Comorbid medical history or use of medications which, in the opinion of the investigators, will interfere with the study procedures or analysis, or compromise participant safety

\section{Outcome Measures}

Alcohol misuse was assessed using the AUDIT and SMAST-G alongside blood samples taken for carbohydrate deficient transferring (CDT) analysis. Routine Liver Function Tests (LFTs) were conducted; albumin, alanine transaminase (ALT), alkaline phosphatase (ALP), bilirubin, and additionally gamma-glutamyl transferase (GGT) and mean corpuscular volume (MCV) for which abnormal levels are both linked to alcohol misuse [47]. The adapted proxy AUDIT was taken to assess carer's opinion of patient's alcohol use.

Cognition was reassessed on day of testing using the Addenbrooke's Cognitive Examination - Revised (ACE-R). The ACE-R has two validated cut off points that were used to confirm cognitive impairment [44]. The MMSE, a measure of cognitive function originally designed as a screening tool for dementia, can be obtained from the ACE-R and was used as per NICE Guidelines as a confirmation of cognitive impairment (A score of 25-30 is considered normal, 21-24 as mild impairment, $10-20$ as moderate and $<10$ as severe cognitive impairment) [48].

Quality of life was assessed using the EQ-5D-3L for the patient, and the proxy EQ-5D-3L for the carer to assess their opinion of subject's general health state [49]. In addition, the Neuropsychiatric Inventory (NPI) was conducted to assess carer's view of the patient's behaviour and possible neuropsychiatric symptoms [50].

\section{Analysis}

Data was entered in SPSS (IBM 24). Multi-modal analysis examined the relationship between self-report AUDIT, proxy AUDIT and SMAST-G ratings and CDT levels. The relationship between alcohol misuse and level of cognitive impairment and quality of life ratings was explored.

\section{Results}

Sixty one subjects were recruited ( 38 male and 23 female) between the ages of 63 and 90 (mean age $=77.85 \mathrm{yrs}$ ). There was no significant difference in age, ACE-R, MMSE, "proxy" AUDIT or CDT values between males and females. However, there was a higher AUDIT score amongst males than females in our sample (Table 1). 
Table 1: There was no significant difference $(p>0.05)$ in age, ACE-R, MMSE, AUDIT, “Proxy” AUDIT or CDT values between males and females

\begin{tabular}{|c|c|c|c|c|c|c|}
\hline & Gender & $\mathrm{N}$ & Mean & $\begin{array}{c}\text { Standard } \\
\text { Deviation }\end{array}$ & $\begin{array}{c}\text { Standard } \\
\text { Error } \\
\text { Mean }\end{array}$ & Significance \\
\hline \multirow{2}{*}{ AGE } & M & 38 & 78.21 & 6.99 & 1.13 & \multirow{2}{*}{$\begin{array}{l}\mathrm{t}=-0.527 \\
\mathrm{p}=0.600\end{array}$} \\
\hline & $\mathrm{F}$ & 23 & 77.26 & 6.53 & 1.36 & \\
\hline \multirow{2}{*}{ ACE-R } & $M$ & 38 & 62.66 & 17.12 & 2.78 & \multirow{2}{*}{$\begin{array}{c}\mathrm{t}=-0.440 \\
\mathrm{p}=0.662\end{array}$} \\
\hline & $\mathrm{F}$ & 22 & 64.91 & 22.21 & 4.74 & \\
\hline \multirow{2}{*}{ MMSE } & $\mathrm{M}$ & 38 & 20.50 & 5.88 & 0.95 & \multirow{2}{*}{$\begin{array}{c}\mathrm{t}=-0.187 \\
\mathrm{p}=0.852\end{array}$} \\
\hline & $\mathrm{F}$ & 22 & 20.82 & 7.12 & 1.52 & \\
\hline \multirow{2}{*}{ AUDIT } & M & 38 & 3.71 & 3.38 & 0.55 & \multirow{2}{*}{$\begin{array}{l}\mathrm{t}=0.762 \\
\mathrm{p}=0.449\end{array}$} \\
\hline & $\mathrm{F}$ & 23 & 3.09 & 2.56 & 0.53 & \\
\hline \multirow{2}{*}{$\begin{array}{l}\text { "Proxy" } \\
\text { AUDIT }\end{array}$} & M & 37 & 3.89 & 3.85 & 0.63 & \multirow{2}{*}{$\begin{array}{l}\mathrm{t}=-0.985 \\
\mathrm{p}=0.329\end{array}$} \\
\hline & $\mathrm{F}$ & 23 & 3.04 & 1.87 & 0.39 & \\
\hline \multirow{2}{*}{ CDT } & M & 31 & 0.68 & 0.21 & 0.04 & \multirow{2}{*}{$\begin{array}{l}\mathrm{t}=0.624 \\
\mathrm{p}=0.535\end{array}$} \\
\hline & $\mathrm{F}$ & 19 & 0.64 & 0.21 & 0.05 & \\
\hline
\end{tabular}

All of the patients and carers who consented completed all aspects of data collection, except 11 whose blood samples were not obtained. $70.5 \%$ (43) were married or living with a life partner and $18 \%$ (11) lived on their own. The mean MMSE and ACE-R scores were $20.62(\mathrm{sd}=6.3)$ and $63.48(\mathrm{sd}=18.9)$ respectively, but there was no correlation with age [MMSE-Age: $r=-0.174(\mathrm{p}=0.183)$, ACE-R-Age: $\mathrm{r}=-0.166(\mathrm{p}=0.206)]$ or years of education [MMSE-education: $\mathrm{r}=$ $0.219(\mathrm{p}=0.114)$, ACE-R-education: $\mathrm{r}=0.178(\mathrm{p}=0.202)]$.

Within the 61 participants, the mean frequency of alcohol use was two to four times per month (Figure 1) and the AUDIT scores ranged from 0 to 15 (Figure 2).

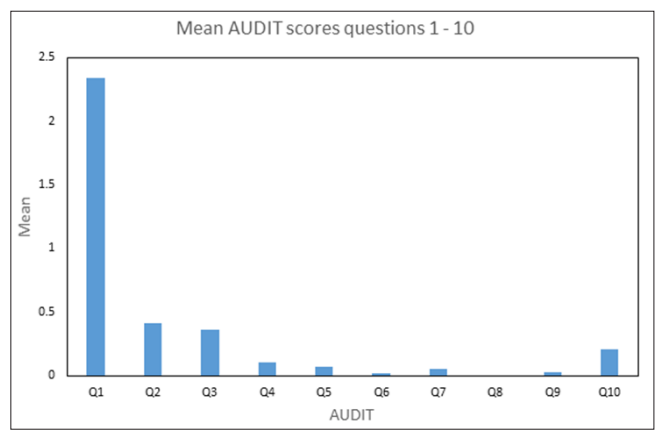

Figure 1: 11 participants were asked to report on their alcohol consumption via the AUDIT questionnaire. This table shows the mean scores on each subquestion, suggesting a mean frequency of alcohol use of two-four times per month.

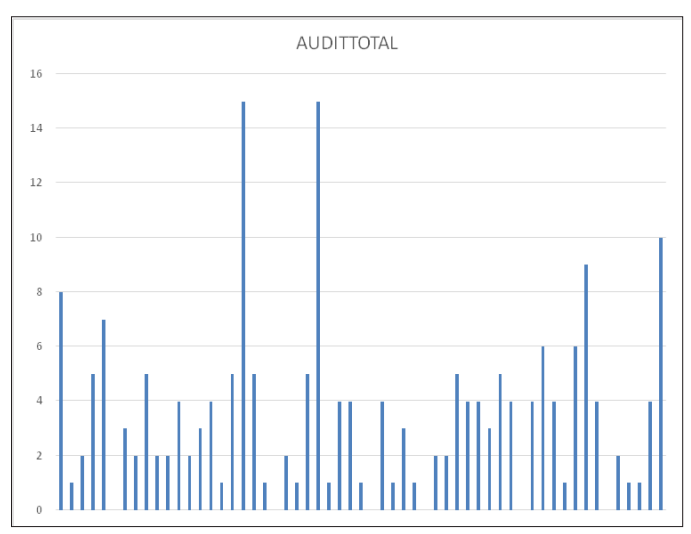

Figure 2: Individual AUDIT scores per participant

The mean AUDIT and proxy AUDIT scores were $3.48(\mathrm{sd}=3.08)$ and $3.27(\mathrm{sd}=3.24)$ respectively, and were significantly correlated $\mathrm{r}=0.666(\mathrm{p}=0.000)$.

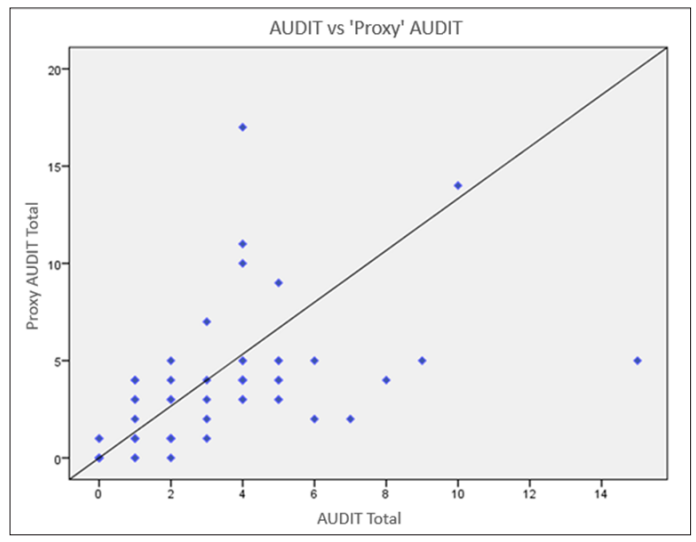

Figure 3: AUDIT vs "Proxy" AUDIT scores.

Of the questions posed in the AUDIT and "proxy" AUDIT, the highest correlation was on the first question, which asks how often the subject drinks alcohol $(\mathrm{r}=0.708, \mathrm{p}=0.000)$. The S-MAST score correlated significantly with the AUDIT score $(r=0.318, p=0.013)$.

The mean blood plasma CDT level was $0.666 \%(\mathrm{sd}=0.21)$ and was not elevated in any subjects (normal range $<1.6 \%$ ). There was no correlation between CDT levels and the AUDIT $(r=-0.041, \mathrm{p}=0.777)$. The negative correlation between the CDT and the AUDIT is interesting given both variables are a measure of alcohol consumption, but is probably a reflection of the fact that we recruited predominantly mild drinkers. There was no significant correlation between CDT and liver function tests (albumin, ALT, ALP), bilirubin, GGT or MCV levels. There was a significant correlation between the AUDIT score and bilirubin $(\mathrm{r}=-0.348, \mathrm{p}=0.012)$, ALP $(\mathrm{r}=-0.280$, $\mathrm{p}=0.040)$ and $\mathrm{MCV}(\mathrm{r}=0.325, \mathrm{p}=0.026)$ levels. The "proxy" AUDIT score significantly correlated with the MCV level $(\mathrm{r}=0.316, \mathrm{p}=0.032)$ and also the ALP level $(r=0.284, p=0.039)$.

The "proxy" AUDIT score versus the plasma CDT level did not show any significant correlation $(\mathrm{r}=0.068, \mathrm{p}=0.640)$. The positive correlation between the CDT and "proxy" AUDIT is in contrast to the negative correlation between CDT and the AUDIT, possibly suggesting that the "proxy" AUDIT could be an even more accurate alcohol misuse measure than the AUDIT, however this conclusion 
must be taken very tentatively given the lack of significance.

We tested several cut off points for the AUDIT; 4,6,8 to ascertain if this altered the correlation with liver function tests. The significant correlation with MCV was maintained in all test groups $(\mathrm{r}=0.429$, $\mathrm{p}=0.008, \mathrm{r}=0.432, \mathrm{p}=0.004$ and $\mathrm{r}=0.432, \mathrm{p}=0.004$ respectively), but there were no other significant correlations with the other LFTs. This was also the case with the "proxy" AUDIT $(r=0.406, p=0.014, r=0.311$, $\mathrm{p}=0.048$ and $\mathrm{r}=0.349, \mathrm{p}=0.023$ respectively), but in this case the ALP level was also significantly correlated at the 6 and 8 cut off level $(\mathrm{r}=-0.370 \mathrm{p}=0.009$ and $\mathrm{r}=-0.350, \mathrm{p}=0.013)$. There were 11 subjects without CDT levels. In this subgroup, the mean AUDIT and proxy AUDIT scores were $3.64(\mathrm{sd}=2.91)$ and $4.91(\mathrm{sd}=4.44)$ respectively.

There was a significant correlation between the AUDIT and ACE-R scores $(\mathrm{r}=0.264, \mathrm{p}=0.041)$, but not the "proxy" AUDIT and the ACE-R ( $r=0.024, p=0.860)$, suggesting no clear trend of alcohol use with cognitive score.

The mean score for the NPI was low, at 15.3 ( $\mathrm{sd}=17.21)$, with no correlation between the NPI and the AUDIT( $\mathrm{r}=0.110, \mathrm{p}=0.408)$. The mean score for the EQ-5D-3L overall health state was 74.9 $(\mathrm{sd}=18.18)$. The mean score for the proxy EQ-5D-3L overall health state was $62.3(\mathrm{sd}=22.52)$, with significant correlation between the participant's and the carer's scores $(\mathrm{r}=0.408, \mathrm{p}=0.001)$. There was no correlation between the EQ-5D-3L overall health state and the $\operatorname{AUDIT}(\mathrm{r}=0.022, \mathrm{p}=0.865)$ or the ACE-R $(\mathrm{r}=0.010, \mathrm{p}=0.938)$. There was a positive trend, though not significant correlation, between the NPI and the EQ-5D-3L $(r=0.245, \mathrm{p}=0.062)$.

\section{Discussion}

Our research aim was driven by a lack of robust data on the validity of screening instruments for alcohol consumption in the cognitively impaired elderly. This study was the first to look at the validity of a proxy measure of alcohol intake in elderly people with cognitive impairment and poses an interesting alternative for primary alcohol screening in MCI and dementia. Our finding that males scored higher on the AUDIT replicates previous literature, which has shown that females tend to score lower on the AUDIT and therefore need lower cut off points to achieve the same sensitivity and specificity as males [51]. However, the significant difference in gender scores of the AUDIT in our sample could be due to females under-reporting their alcohol consumption compared to males, or males over-estimating their consumption.

Our results suggest that the AUDIT may be a valid screening tool for the assessment of alcohol misuse in an elderly population with cognitive impairment. There was no correlation between the AUDIT score and CDT level, with CDT remaining fairly constant over a range of AUDIT scores. This suggests that the AUDIT may not be a useful tool in identifying alcohol misuse in the elderly with CI, however we need to be cautious about this assumption, as our sample was relatively small and skewed towards lower alcohol consumption. This finding is in contrast to the AUDITs previous validation in an elderly population without CI [32]. Alternatively, the lack of correlation between the AUDIT and CDT level could also reflect on the sensitivity of the CDT in an elderly population. No participant had a raised CDT level possibly suggesting that the CDT level is not sensitive enough to pick up any cases of alcohol misuse in our study sample despite participants being AUDIT and "proxy" AUDIT positive. Moreover, some patients had elevated
LFTs, MCV and GGT which could suggest that these biomarkers, although not specific for alcohol misuse could be more sensitive in the elderly in picking up alcohol misuse. An example of this from our data was the significant correlation between the AUDIT and bilirubin, ALP and MCV levels.

The "proxy" AUDIT was no more efficient at assessing alcohol intake than the AUDIT when compared to the subject's CDT level, suggesting that carers could be equally unreliable in assessing alcohol intake in our sample. The slightly positive correlation between the "proxy" AUDIT and the CDT could indicate some level of validity compared to the AUDIT, however as this was not a significant finding, more data is needed in order to comment more confidently on the validity of this screening tool. The lack of difference between the AUDIT and "proxy" AUDIT could be due to a very small number of drinkers in our sample size. It would seem likely that any discrepancy in report of alcohol consumption would be associated with actual alcohol consumption. Graham and Jackson found that participants and proxies were 4.3 times more likely to agree on alcohol consumption if the participants were never or infrequent drinkers [40]. This suggests that the "proxy" AUDIT should be tested in a much larger sample of carers of cognitively impaired older people who are misusing alcohol, in order to be confident that a carer's report is no more or less accurate than a self-report of alcohol intake using the AUDIT. The similarity in report could also be due to subject misreporting their alcohol use from fear of judgement or simply a lack of adequate recall, and carer misreporting from embarrassment, possibly if they feel responsible for their relative's alcohol intake. In addition, comparing the validity of the AUDIT in individuals with MCI compared to age-matched healthy controls would provide further information as to whether this is a useful screening tool in this population [51].

Overall health rating from 0 to 100 on the EQ-5D 3L was higher than the proxy EQ-5D 3L overall health rating (although the difference was not significant), suggesting that carers view the quality of the subjects' lives as lower than the subjects. Reasons for this could include subjects making a skewed judgement of their quality of life (QoL) because of their level of dementia, or that carers could find it hard to rate the subject's quality of life highly given their cognitive deficits. Moreover, the carers' answers could be influenced by their own QoL, which may be reduced in part from carers' burden. In fact, this projection of QoL has been identified with the EQ-5D previously, where Arons found that proxy scoring of their relative's QoL reflected the rater's own quality of life [52]. Quality of life, measured by self-report or proxy, was not associated with alcohol consumption in our sample, suggesting that levels of consumption did not affect the subjects' perception of QoL. However, it could also be due to the fact that no alcohol misusers (as measured by the CDT) were identified in our sample and therefore not enough subjects drank enough to affect their QoL negatively. The low NPI scores and the trend for these to reflect the EQ-5D scores suggest a low level of behavioural symptoms in the sample group.

The "Our Invisible Addicts" report suggested that the number of people misusing alcohol in the over 40 age group has increased, and as this cohort ages we should anticipate an increase in elderly people misusing alcohol. Given also, the increase in prevalence of cognitive impairment in the elderly, it seems likely that there will be a larger proportion of the older population with both cognitive impairment and alcohol misuse in the future. It is therefore vital that we are 
well equipped to be able to identify those who may be excessively drinking. Kaner found that population screening for harmful and hazardous alcohol use, followed by brief intervention, could result in population-based reductions in alcohol consumption, highlighting that we have the ability to reduce the harm alcohol does to the population if we can successfully identify those at risk [53]. It has been previously shown that alcohol-related dementia is associated with an increased length of hospital stay, further highlighting the importance of reducing alcohol consumption in this population [54].

Our study limitations include a small sample size from a selective population presenting with memory difficulties to a specialist outpatient clinic. Additional studies are needed to further examine the validity of the AUDIT and "proxy" AUDIT in older patients with CI and their carers respectively, in order to be confident of our findings. Furthermore, using the AUDIT in a proxy manner when it has been designed for self-report may introduce a degree of inaccuracy, as carers may not be able to answer certain questions designed to be answered by the participant, or simply, a carer may not know the answer to a question about the participant's alcohol consumption, which is not currently a viable option in the scoring of the AUDIT. Although, in general, most carers could answer confidently on the subject's alcohol consumption, it could also be affected by the number of hours they spent with the subject per week. As we did not record time spent with the subject, this could be a confounding factor in the agreement of alcohol consumption between subject and carer, as the carer's report could be more accurate if they spend more time with the subject. Moreover, although all study investigators received training in administering the AUDIT, SMAST-G, ACE-R, EQ-5D and NPI, interviewer bias may have been introduced if one interviewer was able to illicit a more honest alcohol history than another. Given the small sample size, this could potentially be a significant bias. In addition, some interviewers were not blinded to the AUDIT score when interviewing the carer using the "proxy" AUDIT, which could introduce an additional bias. Furthermore, we intended to examine patients with mild cognitive impairment or mild dementia, but our sample was skewed to the mild dementia range. In addition, we did not restrict our sample to any particular dementia diagnosis, both factors which may have skewed our results. Further studies should include larger sample sizes with a broader range of cognitive impairment, to allow the acquisition of prevalence data of alcohol misuse in this group and categorise the associations of alcohol with different aetiologies and severities of cognitive impairment and dementia.

We found no association between alcohol consumption and cognition in our population, again indicating that a larger sample size may be needed in order to be able to detect any association. Further research would also need to include longitudinal follow up studies in order to assess the effects of alcohol intake on the development and/or progression of CI to dementia, and whether early interventions could make a difference to morbidity or mortality associated with alcohol misuse. Lopes found that moderate alcohol consumption could be protective against the development of CI when compared to non-drinkers and heavy drinkers [55]. In addition, $\mathrm{Xu}$ found a similar association between alcohol consumption and progression from mild cognitive impairment to dementia [56]. They found a J-shaped relationship with slightly increased progression to dementia of abstainers, and the greatest increase in progression among heavy drinkers compared to moderate consumers of alcohol. However, Almeida suggested that alcohol does not have a direct impact of cognitive impairment in later life through a Mendelian randomisation six year follow-up study of alcohol de hydrogenase variants. Although conversely, in agreement with Lopes and $\mathrm{Xu}$, they suggested that moderate consumption may be protective against cognitive impairment in men[15,55-56].

Although the literature appears to support the protective role of moderate levels of alcohol consumption regarding cognitive impairment, Cooper found that moderate lifetime consumption of alcohol does not affect levels of cognition in the elderly in their study of almost 2000 patients aged 60-74 years [57]. They found that premorbid education, a lifestyle of moderation and decreased drinking in those who had physical illnesses confounded results. Therefore, it seems the effects of alcohol consumption on cognition in the elderly remain unclear. However, what is apparent is that alcohol consumption and cognitive impairment are on the rise in our elderly population, which poses many questions that need addressing. Does alcohol consumption affect cognition in the elderly? If so, can we accurately identify those at risk? And can we then implement suitable policy or guidelines to possibly prevent or delay progression of cognitive impairment which will have sufficient impact on public health outcomes?

\section{Conclusion}

Our data suggests that the AUDIT and the "proxy" AUDIT maybe valid screening tools in an elderly population with cognitive impairment and their careers, however, a larger, more representative sample size is needed before firm conclusions can be drawn from our study. Moreover, given the lack of positive CDT findings in our sample, further studies are needed to evaluate the sensitivity and specificity of this biomarker in the elderly as other more commonly used biomarkers may be better screening tests in this population. A recent study has evaluated the benefit of the CDT to detect chronic alcohol use in the elderly, and has noted that most alcohol dependence diagnosis tools are based on research of younger populations [58]. Results of this study are yet to be published.

\section{Ethical Considerations}

This study has been approved by the NRES Committee South East Coast. All participation was voluntary, with informed consent.

\section{References}

1. Psychiatrists, R.C.o (2011) Our invisible addicts: First report of the Older Persons' Substance Misuse Working Group of the Royal College of Psychiatrists. Royal College of Psychiatrists.

2. McManus S, Meltzer H Brugha, TS Bebbington, PE Jenkins R (2009) Adult psychiatric morbidity in England, 2007: results of a household survey. The NHS Information Centre for health and social care.

3. Drummond C, Oyefeso N, Phillips T, Cheeta Survjit, Deluca Paolo (2004) Alcohol needs assessment research project (ANARP). The national needs assessment for England. London: Department of Health and the National Treatment Agency.

4. Moos RH, Schutte KK, Brennan PL, Moos BS (2009) Older adults' alcohol consumption and late-life drinking problems: a 20-year perspective. Addiction 104: 1293-1302.

5. Sorocco KH, SW Ferrell (2006) Alcohol use among older adults. The Journal of general psychology 133: 453-467.

6. Kalla A, V.M Figueredo (2017) Alcohol and cardiovascular disease in the geriatric population. Clin Cardiol 40: 444-449.

7. Duru OK, Xu H, Tseng CH, Mirkin M, Ang A et al. (2010) Cor- 
relates of Alcohol-Related Discussions Between Older Adults and Their Physicians. Journal of the American Geriatrics Society 58: 2369-2374.

8. Kalant H, Gomberg E, Hegedius A, Zucker (1998) Pharmacological interactions of aging and alcohol. Alcohol problems and aging. NIAAA Research Monograph 99-116.

9. St John PD, WM Snow, SL Tyas (2010) Alcohol use among older adults. Reviews in Clinical Gerontology 20: 56-68.

10. Busse A, Bischkopf J, Riedel-Heller SG, Angermeyer MC (2003) Sub classifications for mild cognitive impairment: prevalence and predictive validity. Psychological medicine 33: 1029-1038.

11. Mitchell AJ, M. Shiri-Feshki (2009) Rate of progression of mild cognitive impairment to dementia-meta-analysis of 41 robust inception cohort studies. Acta Psychiatrica Scandinavica 119: 252-265.

12. Randall-James J, Wadd S, Edwards K, Thake A (2015) Alcohol screening in people with cognitive impairment: an exploratory study. Journal of dual diagnosis 11: 65-74.

13. Wadd S, J Randall, A Thake, K Edwards, S Galvanil et al. (2013) Alcohol misuse and cognitive impairment in older people: An exploratory study.

14. S Sabia, Alexis Elbaz, Annie Britton, Steven Bell, Aline Dugravot et al. (2014) Alcohol consumption and cognitive decline in early old age. Neurology 82: 332-339.

15. Almeida OP, Hankey GJ, Yeap BB, Golledge J, Flicker L (2014) Alcohol consumption and cognitive impairment in older men A mendelian randomization study. Neurology 82: 1038-1044.

16. Langballe EM, Ask H, Holmen J, Stordal E, Saltvedt I et al. (2015) Alcohol consumption and risk of dementia up to 27 years later in a large, population-based sample: the HUNT study, Norway. European journal of epidemiology 30: 1049-1056.

17. Mukamal KJ, Kuller LH, Fitzpatrick AL, Longstreth WT Jr, Mittleman MA et al. (2003) Prospective study of alcohol consumption and risk of dementia in older adults. Jama 289: 1405-1413.

18. Sachdeva A, Mina Chandra, Mona Choudhary, Prabhoo Dayal, Kuljeet Singh Anand (2016) Alcohol-related dementia and neurocognitive impairment: a review study. International journal of high risk behaviours \& addiction 5(3).

19. Peters R, Peters J, Warner J, Beckett N, Bulpitt C (2008) Alcohol, dementia and cognitive decline in the elderly: a systematic review. Age and ageing 37: 505-512.

20. Chan KKK, KC Chiu, LW Chu (2010) Association between alcohol consumption and cognitive impairment in Southern Chinese older adults. International journal of geriatric psychiatry $25: 1272-1279$.

21. Atkinson RM (2002) Substance abuse in the elderly, in Psychiatry in the Elderly, O.C. Jacoby R, Editor. Oxford University Press 799-834.

22. Gupta S, J. Warner (2008) Alcohol-related dementia: a 21st-century silent epidemic? RCP.

23. Tan JE, BA. Springate G. Tremont (2012) Neuropsychologists' beliefs about alcohol and dementia. The Clinical Neuropsychologist 26: 879-893.

24. Mackinnon A, R. Mulligan (1998) Combining cognitive testing and informant report to increase accuracy in screening for dementia. American Journal of Psychiatry 155: 1529-1535.

25. Mackinnon A, Khalilian A, Jorm AF, Korten AE, Christensen $\mathrm{H}$ et al. (2003) Improving screening accuracy for dementia in a community sample by augmenting cognitive testing with informant report. Journal of Clinical Epidemiology 56: 358-366.
26. Nielsen TR, Phung TK, Chaaya M, Mackinnon A, Waldemar G (2016) Combining the Rowland universal dementia assessment scale and the informant questionnaire on cognitive decline in the elderly to improve detection of dementia in an Arabic-speaking population. Dementia and geriatric cognitive disorders 41: 46-54.

27. Health, N.C.C.f.M., N.I.f. Health, and C. Excellence (2011) Alcohol use disorders: The NICE Guideline on the Diagnosis, Assessment and Management of Harmful Drinking and Alcohol Dependence. RCPsych Publications.

28. Saunders JB, Aasland OG, Babor TF, de la Fuente JR, Grant M (1993) Development of the alcohol use disorders identification test (AUDIT): WHO collaborative project on early detection of persons with harmful alcohol consumption-II. Addiction 88: 791-804.

29. Bohn MJ, TF Babor, HR Kranzler (1995) The Alcohol Use Disorders Identification Test (AUDIT): validation of a screening instrument for use in medical settings. Journal of studies on alcohol 56: 423-432.

30. Steinbauer JR, Cantor SB, Holzer CE, Volk RJ (1998) Ethnic and sex bias in primary care screening tests for alcohol use disorders. Annals of Internal Medicine 129: 353-362.

31. Volk RJ, Steinbauer JR, Cantor SB, Holzer CE (1997) The Alcohol Use Disorders Identification Test (AUDIT) as a screen for at-risk drinking in primary care patients of different racial/ ethnic backgrounds. Addiction 92: 197-206.

32. Berks J, R. McCormick (2008) Screening for alcohol misuse in elderly primary care patients: a systematic literature review. International Psycho geriatrics 20: 1090-1103.

33. Aalto M, Alho H, Halme JT, Seppä K (2011) The alcohol use disorders identification test (AUDIT) and its derivatives in screening for heavy drinking among the elderly. International journal of geriatric psychiatry 26: 881-885.

34. Ryou YI, Kim JS, Jung JG, Kim SS, Choi DH (2012) Usefulness of alcohol-screening instruments in detecting problem drinking among elderly male drinkers. Korean journal of family medicine 33: 126-133.

35. Fiellin DA, M.C Reid, PG. O'connor (2000) Screening for alcohol problems in primary care: a systematic review. Archives of internal medicine 160: 1977-1989.

36. Blow F, Brower K.J, Schulenberg JE, Demo-Dananberg LM, Young JP et al. (1992) The Michigan alcoholism screening test-geriatric version (MAST-G): a new elderly-specific screening instrument. Alcohol Clin Exp Res 16: 372.

37. Hirata ES, Almeida OP, Funari RR, Klein EL (2001) Validity of the Michigan Alcoholism Screening Test (MAST) for the Detection of Alcohol-Related Problems Among Male Geriatric Outpatients. The American Journal of Geriatric Psychiatry 9: 30-34.

38. Blow F (1998) Brief screening for alcohol problems in elderly populations using the Short Michigan Alcoholism Screening Test-Geriatric Version (SMAST-G). Alcoholism: Clinical and Experimental Research 22: 131.

39. Walter A Rocca, Laura Fratiglioni, Laura Bracco, Daniela Pedone, Cinzia Groppi et al. (1986) The use of surrogate respondents to obtain questionnaire data in case-control studies of neurologic diseases. Journal of chronic diseases 39: 907-912.

40. Graham P, R Jackson (1993) Primary versus proxy respondents: comparability of questionnaire data on alcohol consumption. American journal of epidemiology 138: 443-452.

41. Gjerde H, Johnsen J, Bjørneboe A, Bjørneboe GE, Mørland J 
(1988) A comparison of serum carbohydrate-deficient transferring with other biological markers of excessive drinking. Scandinavian journal of clinical and laboratory investigation 48: 1-6.

42. Kapur A, G Wild, A Milford-Ward, D R Triger (1989) Carbohydrate deficient transferring: a marker for alcohol abuse. BMJ 299: 427-431.

43. Donovan DM, Dunn CW, Rivara FP, Jurkovich GJ, Ries RR et al. (2004) Comparison of trauma centre patient self-reports and proxy reports on the Alcohol Use Identification Test (AUDIT). J Trauma 56: 873-82.

44. Mioshi E, Dawson K, Mitchell J, Arnold R, Hodges JR (2006) The Addenbrooke's Cognitive Examination Revised (ACE-R): a brief cognitive test battery for dementia screening. International journal of geriatric psychiatry 21: 1078-1085.

45. Claire AG Wolfs, Carmen D Dirksen, Alfons Kessels, Daniëlle CM Willems, Frans RJ Verhey et al. (2007) Performance of the EQ-5D and the EQ-5D+ C in elderly patients with cognitive impairments. Health and quality of life outcomes 5: 33.

46. Cummings JL, Mega M, Gray K, Rosenberg-Thompson S, Carusi DA et al. (1994) The Neuropsychiatric Inventory comprehensive assessment of psychopathology in dementia. Neurology 44: 2308-2308.

47. Albertini A, Bonera E, Proto C, Grasso Caprioli G (1973) Proceedings: Correlation between gamma-glutamyl transpeptidase serum levels and liver damage due to alcohol. Quaderni Sclavo di diagnostica clinica e di laboratorio 9: 277-284.

48. Folstein MF, SE Folstein, P.R. McHugh (1975) "Mini-mental state": a practical method for grading the cognitive state of patients for the clinician. Journal of psychiatric research 12: 189-198.

49. Dolan P (1997) Modeling valuations for EuroQol health states. Medical care 35: 1095-1108.

50. Kaufer DI, Cummings JL, Ketchel P, Smith V, MacMillan A et al. (2000) Validation of the NPI-Q, a brief clinical form of the Neuropsychiatric Inventory. The Journal of neuropsychiatry and clinical neurosciences 12: 233-239.
51. Neumann T, Neuner B, Gentilello LM, Weiss-Gerlach E, Mentz $\mathrm{H}$ et al. (2004) Gender differences in the performance of a computerized version of the alcohol use disorders identification test in sub critically injured patients who are admitted to the emergency department. Alcoholism: Clinical and Experimental Research 28: 1693-1701.

52. Arons AM, Krabbe PF, Schölzel-Dorenbos CJ, van der Wilt GJ, Rikkert MG (2013) Quality of life in dementia: a study on proxy bias. BMC medical research methodology 13: 110.

53. Kaner EF, Beyer F, Dickinson HO, Pienaar E, Campbell F et al. (2007) Effectiveness of brief alcohol interventions in primary care populations. The Cochrane Library.

54. Draper B, Karmel R, Gibson D, Peut A, Anderson P (2011) Alcohol-related cognitive impairment in New South Wales hospital patients aged 50 years and over. Australian \& New Zealand Journal of Psychiatry 45: 985-992.

55. Lopes MA, Furtado EF, Ferrioli E, Litvoc J, Bottino CM (2010) Prevalence of Alcohol-Related Problems in an Elderly Population and Their Association With Cognitive Impairment and Dementia. Alcoholism: Clinical and Experimental Research 34: 726-733.

56. Xu G, Liu X, Yin Q, Zhu W, Zhang R et al. (2009) Alcohol consumption and transition of mild cognitive impairment to dementia. Psychiatry and clinical neurosciences 63: 43-49.

57. Cooper C, Bebbington P, Meltzer H, Jenkins R, Brugha T et al. (2009) Alcohol in moderation, premorbid intelligence and cognition in older adults: results from the Psychiatric Morbidity Survey. Journal of Neurology, Neurosurgery \& Psychiatry 80: 1236-1239.

58. Benefit of Carbohydrate Deficient Transferring to Detect Chronic Alcohol Abuse in the Elderly. A French Multicentered Prospective Interventional Study.; Available from: https://clinicaltrials.gov/ct2/show/NCT02822911.
Copyright: (C2018 Vanessa Raymont, et al. This is an open-access article distributed under the terms of the Creative Commons Attribution License, which permits unrestricted use, distribution, and reproduction in any medium, provided the original author and source are credited. 